\title{
\begin{tabular}{l|l} 
Mibraries & DSpace@MIT
\end{tabular}
}

\author{
MIT Open Access Articles
}

Framework for the analysis of the adaptability, extensibility, and scalability of semantic information integration and the context mediation approach

The MIT Faculty has made this article openly available. Please share how this access benefits you. Your story matters.

Citation: Gannon, T. et al. "Framework for the Analysis of the Adaptability, Extensibility, and Scalability of Semantic Information Integration and the Context Mediation Approach." System Sciences, 2009. HICSS '09. 42nd Hawaii International Conference on. 2009. 1-11. (c2009 Institute of Electrical and Electronics Engineers.

As Published: http://dx.doi.org/10.1109/HICSS.2009.223

Publisher: Institute of Electrical and Electronics Engineers

Persistent URL: http://hdl.handle.net/1721.1/59324

Version: Final published version: final published article, as it appeared in a journal, conference proceedings, or other formally published context

Terms of Use: Article is made available in accordance with the publisher's policy and may be subject to US copyright law. Please refer to the publisher's site for terms of use. 


\section{Framework for the Analysis of the Adaptability, Extensibility, and Scalability of Semantic Information Integration and the Context Mediation Approach}

\author{
Thomas Gannon \\ MITRE Corporation \\ tgannon@mitre.org \\ Michael Siegel \\ MIT Sloan School of \\ Management \\ msiegel@mit.edu
}

\author{
Stuart Madnick \\ MIT Sloan School of \\ Management \& \\ MIT School of Engineering \\ smadnick@mit.edu \\ Marwan Sabbouh \\ MITRE Corporation \\ ms@mitre.org
}

\author{
Allen Moulton \\ MIT Sloan School of \\ Management \\ amoulton@mit.edu \\ Hongwei Zhu \\ Old Dominion University \\ hzhu@odu.edu
}

\begin{abstract}
Technological advances such as Service Oriented Architecture (SOA) have increased the feasibility and importance of effectively integrating information from an ever widening number of systems within and across enterprises. A key difficulty of achieving this goal comes from the pervasive heterogeneity in all levels of information systems. A robust solution to this problem needs to be adaptable, extensible, and scalable. In this paper, we identify the deficiencies of traditional semantic integration approaches. The COntext INterchange (COIN) approach overcomes these deficiencies by declaratively representing data semantics and using a mediator to create the necessary conversion programs from a small number of conversion rules. The capabilities of COIN is demonstrated using an example with 150 data sources, where COIN can automatically generate the over 22,000 conversion programs needed to enable semantic interoperability using only six parametizable conversion rules. This paper presents a framework for evaluating adaptability, extensibility, and scalability of semantic integration approaches. The application of the framework is demonstrated with a systematic evaluation of COIN and other commonly practiced approaches.
\end{abstract}

\section{Introduction}

In the report, "Making the Nation Safer", the National Research Council [17] found that "Although there are many private and public databases that contain information potentially relevant to counter terrorism programs, they lack the necessary context definitions (i.e., metadata) and access tools to enable interoperation with other databases and the extraction of meaningful and timely information." Despite the fact that nearly $30 \%$ of IT dollars are spent on Enterprise Information Integration (EII), organizations are still plagued by the lack of effective integration and interoperation. NIST found that lack of interoperability costs the U.S. capital facilities industry $\$ 15.8$ billion per year [8]. As society and enterprises become increasingly information intensive and the web continues to grow in the range and number of sources, semantic information integration is critical for effective exchange and utilization of valuable information. A viable solution to large scale integration has to be adaptable, extensible, and scalable.

Technologies already exist to overcome heterogeneity in hardware, software, and syntax used in different systems (e.g., the ODBC standard, XMLbased standards, web services and SOA-Service Oriented Architectures.) While these capabilities are essential to information integration, they do not address the issue of heterogeneous data semantics that exist both within and across enterprises. The data receiver still needs to reconcile semantic differences such as converting pounds and ounces into kilograms, or vice versa, depending on how the receiver wants to interpret the data. Hand-coding such conversions is only manageable on a small scale; alternative solutions are needed as the number of systems and the complexity of each system increase.

There have been significant efforts devoted to developing robust semantic integration solutions [20], such as the COntext INterchange (COIN) approach $[2,6,10,11]$. However, it is not until recently that attention is paid on evaluating the robustness of various solutions. Two frameworks are provided in [4] 
and [12] to evaluate the capability of a solution. Each framework consists of scenarios with a range of heterogeneous sources and a set of testing queries to see if an integration solution can correctly answer the queries. Neither framework provides criteria for evaluating other important properties of a solution, e.g., adaptability, extensibility, and scalability. These properties, or rather these terms, appear frequently in works that describe certain integration approaches, but they are often used informally. For example, a comparative analysis is given in [14] to compare several integration approaches; certain misinterpretations exist because of a lack of a uniform framework to evaluate these properties.

In contrast, in this paper, we will provide a framework for evaluating adaptability, extensibility, and scalability of semantic integration solutions, and to use the framework to evaluate the COIN approach and several commonly practiced approaches.

\section{Examples and Challenges of Intelligence Information Integration}

As a motivating example, let us consider intelligence information which is usually gathered by different agencies in multiple countries. Since no single agency is expected to have complete information, integration is necessary to perform various intelligence analyses, including basic questions such as "who did what, where, and when". Significant challenges exist when different agencies organize and report information using different conventions. We illustrate the challenges using several examples from the counterterrorism domain. Similar issues exist in most other application domains where information integration is required, especially if heterogeneous semi-structured web sources are involved.

Person Identification: Identifying a person in a corporate database can be as simple as assigning a unique identification number, e.g., employee_id, for each person. This cannot be easily done across multiple agencies. Other attributes of a person are often used to help identify the records related to the person in different data sources.

Name of a person is a good candidate attribute, but different sources may record names differently, e.g., "William Smith" in one source and "Bill Smith" in another. Name spelling becomes more complicated when a foreign name is translated into English. For example, the Arabic name يف has been shown to have over 60 romanizations including: Gadaffi, Gaddafi, Gathafi, Kadafi, Kaddafi, Khadafy, Qadhafi, and Qathafi. There are numerous romanization and transliteration standards. But different agencies may choose different standards.

Other attributes such as weight and height of a person can be used conjunctively to help with identification matching. Again, different agencies may choose different standards for these attributes, e.g., a British agency may report weight in stones, while a U.S. agency might use pounds and a German agency might use kilograms. Similarly, these agencies may use feet, inches, and centimeters, respectively, for height.

It would be impossible to perform any useful intelligence analysis when the information from different sources is put together without reconciling these differences. To illustrate the difficulties, consider three records from three different sources shown in Table 1.

Table 1. Data from three different sources

\begin{tabular}{|l|l|l|l|l|l|l|}
\hline Source & Name & Weight & Height & Place & Time & Event \\
\hline UK & Gadaffi & 12.14 & 5.67 & London & $\begin{array}{l}12 / 11 / 2004 \\
13: 15\end{array}$ & $\begin{array}{l}\text { Plane } \\
\text { arrives }\end{array}$ \\
\hline US & Kadafi & 170 & 68 & London & $\begin{array}{l}11 / 15 / 2004 \\
19: 30\end{array}$ & Meeting \\
\hline Germany & Qadhafi & 77 & 173 & Vienna & $\begin{array}{l}12 / 11 / 2004 \\
11: 30\end{array}$ & $\begin{array}{l}\text { Plane } \\
\text { departs }\end{array}$ \\
\hline
\end{tabular}

In their present form, the three records apparently refer to three different people. However, an important pattern will be revealed when the data from different sources are transformed into a uniform standard. For example, if the three records are converted to the standard used by the U.S. agency, we can relate the three records to the same person because after the conversion, the three records have the same Name, Weight and Height (e.g., 12.14 stones is equal to 179 lbs or $77 \mathrm{~kg}$ ), and discover a pattern that a person named Kadafi, who weighs $170 \mathrm{lbs}$ and measures 68 inches high, flew from Vienna to London on November 12, 2004 and later on November 15, 2004 had a meeting.

Location Representation: Location information is often represented using place names, codes, and various geographic coordinates. Place names are not unique. A search for Cambridge at Weather.com returns eight cities located in Canada, UK, and U.S. Thus it is necessary to qualify a place name with other place names at different geographical granularities, e.g., Cambridge, MA, US or Cambridge, Ontario, CA. Here, country codes are used to indicate the country in which the city is located. Although country codes are compact and can eliminate problems with spelling and translation of country names, the same code sometimes represents different countries in different standards. The frequently used standards include the FIPS 2character alpha codes and the ISO3166 2-character alpha codes, 3-character alpha codes, and 3-digit numeric codes. Confusions will arise when different agencies use different coding standards. For example, 
"explosion heard in the capital of BG" - is it in Bulgaria (if ISO 3166 2-charcter alpha code was used) or in Bangladesh (if FIPS code was used). Similarly, BD stands for Bermuda in FIPS, while it stands for Bangladesh in ISO 3166; and BM stands for Bermuda in ISO 3166 and for Burma in FIPS.

There are also multiple standards for airport codes. The two major ones are IATA and ICAO. For example, the code for Heathrow airport is LHR in IATA standard, EGLL in ICAO standard. A system that uses one code standard will not be able to correctly recognize an airport designated with another standard.

One may contemplate that we should be able to identify a location by its geographical coordinate on earth. That turns out to be very complicated - there are over 40 geographic coordinate systems widely used around the world. Even within the U.S. Department of Defense (DoD) different standards are used by different branches of the armed forces, e.g., parts of the US Army and Marine Corps use the Universal Transverse Mercator (UTM) Grid and Military Grid Reference System (MGRS), while parts of the US Navy use latitude and longitude expressed in degrees, minutes and seconds, and parts of the US Air Force express them in degrees and decimal degrees. Misinterpretation of these different representations can lead to ineffective coordination in the battle field or tactic operations in the war on terror.

Time Representation: The representations for other data elements could vary significantly among data sources. Time representation is particularly important for many applications. For example, date may be expressed using different calendars (e.g., besides the Gregorian calendar, there are others, such as the Jewish/Israeli calendar and Chinese/lunar calendar). Even when only the Gregorian calendar is used, year, month, and day can be arranged in different orders and using different punctuations, e.g., 11/12/2004 versus 12-11-2004, etc.

The time of day values can be at GMT time or local time (with different conventions for how to encode the time zone), standard time or daylight savings time, using either 12-hour or 24-hour format, etc. There is considerable variety of combinations and permutations.

\section{Integration Scenario}

To further illustrate the challenges of integrating information from diverse sources, let us consider a scenario that involves many of the data elements discussed earlier.

After September 11, it became imperative that different agencies in the U.S. and among coalition countries share counter-terrorism intelligence information. Suppose there are a total of 150 such agencies, e.g., two dozen countries each having, on average, half dozen agencies (or possibly different parts of the same agency). Let us assume that the shared information consists of person name, height, weight, airport, country, geo-coordinate of location, date, and time - which, of course, is a small sample of the wide array of information actually used. To further simplify explication, we assume that person name and time data have been standardized across the sources. For the rest of the attributes different agencies may use different conventions. The varieties of these conventions are summarized in Table 2.

Table 2. Semantic Differences in Data Sources

\begin{tabular}{|l|l|}
\hline Data Types & Semantic varieties \\
\hline Height & 4 different units of measure: ft, in, cm, m \\
\hline Weight & 3 different units of measure: lbs, kg, stone \\
\hline Airport & 2 different coding standards: IATA, ICAO \\
\hline Country & $\begin{array}{l}\text { 4 different coding standards: FIPS, ISO 2- } \\
\text { Alpha, ISO 3-Alpha, ISO 3-digit }\end{array}$ \\
\hline $\begin{array}{l}\text { Geo- } \\
\text { coordinate }\end{array}$ & $\begin{array}{l}\text { 4 different reference systems and datum } \\
\text { parameters: MGRS_WGS84, BNG_OGB7, } \\
\text { Geodetic_WGS84,UTM_WGS84 }\end{array}$ \\
\hline Date & $\begin{array}{l}\text { 4 different formats: mm/dd/yyyy, } \\
\text { dd/mm/yyyy, dd.mm.yyyy, dd-mm-yyyy. }\end{array}$ \\
\hline
\end{tabular}

There are a total of 1,536 (i.e., $4 * 3 * 2 * 4 * 4 * 4$ ) combinations from these varieties. We use the term contexts to refer to these different ways of representing and interpreting data - there are potentially 1,536 unique contexts in this scenario. Let us assume that each of the 150 data sources uses a distinct context as its data representation convention. For example, a U.S. agency may choose to use inches for height, lbs for weight, IATA code for airport, etc., while a U.K. agency may choose to use feet for height, stones for weight, ICA for airport, etc.

An analyst from any of the 150 agencies may need information from all the other agencies to perform intelligence analysis. As shown in Table 1, when information from other agencies is not converted into the analyst's context, it will be difficult to identify important patterns. Therefore, a total of 22,350 (i.e., $150 * 149$ ) conversion programs would be required to convert data from any source's context to any other source's context, and vice versa.

In practice, any specific analyst or analytical tool used by the analyst can have a context different from the agency's, e.g., an analyst from the CIA may use a tool that assumes height is in feet while the agency's databases use inches. Therefore, every data source and data receiver could have their own contexts, so that in reality, there can be more than 150 information exchanging entities in the 150 agencies. For explication purposes, we continue the example with the assumption that there are only 150 sources/receivers. 
Implementing tens of thousands of data conversions is not an easy task; but maintaining them to cope with changes in data sources and receiver requirements over time is even more challenging. We will describe and discuss various approaches to this problem in the next two sections.

\section{Traditional Approaches to Achieving Semantic Interoperability}

\begin{abstract}
Brute-force Data Conversions (BF): The BF approach directly implements all necessary conversions in hand-coded programs. With $\mathrm{N}$ data sources and receivers, $\mathrm{N}(\mathrm{N}-1)$ such conversions need to be implemented. When $\mathrm{N}$ is large, these conversions become costly to implement and difficult to maintain. The BF process is labor-intensive because many semantic differences have to be identified by humans and the conversions need to be implemented and maintained over time to account for changes in the underlying sources. This explains why nearly $70 \%$ of integration costs come from the implementation of these data conversion programs [3].

The BF approach might appear sufficiently inefficient that one might be surprised at how common it is. The reason is that conversion programs are usually written incrementally. Each individual conversion program is produced in response to a specific need. Writing "only one conversion program" does not seem like a bad idea, but over time this process leads to $\mathrm{N}(\mathrm{N}-1)$ conversion programs that must be maintained.
\end{abstract}

Global Data Standardization (GS): In the example, different data standards are used in the 150 agencies that need to exchange information. If they could agree on a uniform standard, e.g., standardizing height data to centimeters in all systems, all the semantic differences would disappear and there would be no need for data conversion. Unfortunately, such standardization is usually infeasible in practice for several reasons.

Often there are legitimate needs for storing and reporting data in different forms. For example, while height in centimeters makes sense to an agent in other NATO countries such as Germany, a U.S. agent may not find it useful until it has been converted to feet and inches. Since most integration efforts involve many existing systems, agreeing to a standard often means someone has to change current implementation, which creates disincentives and makes the standard setting and enforcement process extremely difficult. This difficulty is exacerbated when the number of the data elements to be standardized is large. For example, in 1991 the DoD initiated a data administration program that attempted to standardize nearly one million data elements. By the year 2000, DoD had only managed to register 12,000 elements, most of which were infrequently reused. After a decade of costly effort, the DoD realized its infeasibility and switched to an alternative approach to allow different communities of interest to develop their own standards [19].

The change in approach by the DoD manifests the reality of standards development, i.e., there are often competing or otherwise co-existing standards. As seen in the examples in Section 2, there are multiple standards for airport codes and for country codes. Different systems can potentially choose different standards to implement. Thus, in most cases, we cannot hope that semantic differences will be completely standardized away; data conversion is inevitable.

Interchange Standardization (IS): The data exchange parties sometimes can agree on the format of what is to be exchanged, i.e., standardizing a set of concepts as well as interchange formats. The underlying systems do not need store the data according to the standard; it suffices as long as each data sender generates the data according to the standard. Thus each system still maintains its own autonomy. This is different from the global data standardization, where all systems must store data according to a global standard. With $\mathrm{N}$ parties exchanging information, the Interchange Standardization approach requires $2 \mathrm{~N}$ conversions. This is a significant improvement over the brute-force approach that might need to implement conversions between every pair of systems.

This approach has been used for various business transactions, e.g., EDI and various B2B trading standards. In the military setting, the U.S. Message Text Format (MTF) and its NATO equivalent, Allied Data Publication-3, have over 350 standard messages that support a wide range of military operations. This standard has been used for over 50 years and currently an XML version is being developed [16]. As a recent example, the DoD standardized the exchange format of weather related data, which consists of about 1,000 attributes. This standard has been successfully used by several systems that exchange weather data [19]. Similarly, the XML-based Cursor-On-Target (COT) standard, which consists of 3 entities and 13 attributes, has been used successfully by over 40 systems to exchange targeting information [19]. The U.S. Army also succeeded in rapidly integrating a dozen diverse stovepipe battlefield systems using limited scope XML-based interchange standards [9].

Although the IS approach has certain advantages, e.g., local autonomy and a smaller number of conversions required, it also has serious limitations. First, all parties have to have a clear understanding about the domain, decide what data elements should go into the standard, and reach an agreement on the data 
format. This can be costly and time consuming. It took the DoD five years to standardize the weather data interchange format. Furthermore, in many cases it is difficult to foresee what data needs to be exchanged or changes to requirements over time, which makes it inappropriate to have a fixed standard. When the significant information is not specified in the standard, ad-hoc conversions have to be implemented. Lastly, any change to the interchange standard affects all systems and the existing conversion programs.

Summary of Traditional Approaches: Each of the three traditional approaches has certain drawbacks that make them inappropriate for integrating information from a large number of data sources. These weaknesses are summarized below:

- Brute-force data conversions (BF): requires a large number of hand-written conversions that are difficult to maintain;

- Global Data Standardization (GS): it is costly and sometimes impossible to develop a global standard. In addition to legitimate reasons of having multiple standards, there are technological difficulties and organizational resistance for a single standard;

- Interchange Standardization (IS): the standard is static, only suitable for routine data sharing and it still requires a large number of hand-written conversions.

In addition, these approaches lack flexibility to adapt to changes because the data semantics is hard-coded in the conversions for BF, in the standard in GS, and in both the conversions and the standard in the case of IS. A suitable approach needs to overcome these shortcomings. In the next section, we will discuss such an approach that automates code generation for conversions and requires no data standardization.

\section{Ontology-based Context Mediation}

Most of the shortcomings of the traditional approaches can be overcome by declaratively describing data semantics and separating knowledge representation from conversion implementation. There have been a number of research projects that utilize ontology to represent data semantics and to facilitate reconciliation of semantic differences [20]. Since an ontology is essentially an agreement on conceptual models, approaches that require a single, i.e. global, ontology have shortcomings similar to the data standardization approach. Therefore it is desirable to lower or eliminate the reliance on reaching a global agreement on the details of every data element. In the following, we introduce the COntext INterchange (COIN) $[2,6,10,11]$ approach, which allows each data source and receiver to describe its local ontology using a common language and also provides reasoning service to automatically detect and reconcile semantic differences.

\subsection{The COIN Approach}

The COIN approach consists of a deductive objectoriented data model for knowledge representation, a general purpose mediation reasoning service module that determines semantic differences between sources and receivers and generates a mediated query to reconcile them, and a query processor that optimizes and executes the mediated query to retrieve and transform data into user context (see Figure 1).

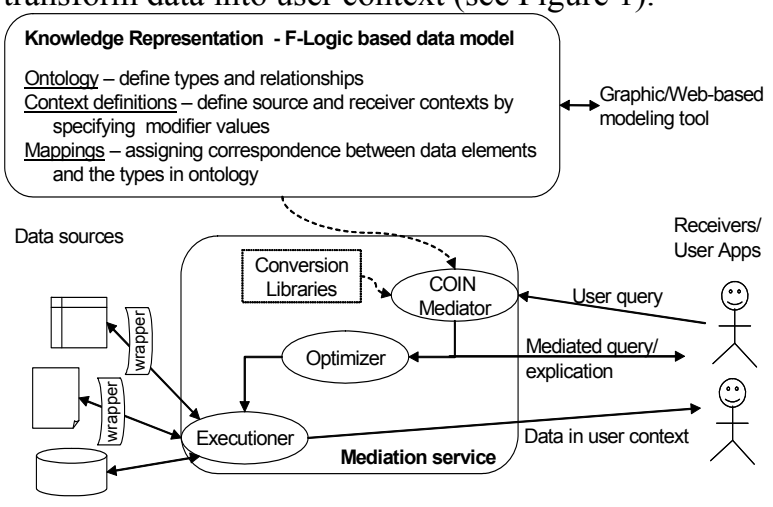

\section{Figure 1. Architecture of COIN System}

The COIN knowledge representation consists of three components. An ontology is used to capture common concepts and their relationships such as one concept being a property (i.e., attribute) or a subconcept (i.e., is_a relationship) of another. A concept is roughly equivalent to a class in object-oriented models and entity type in Entity-Relationship conceptual models. Each concept may have one or more modifiers as a special kind of property to explicitly represent specializations of the concept in the sources and receivers. We call the collection of declarative specifications of modifier values context. For each modifier, a rule or a set of rules are used to specify the conversions between different values of the modifier. The semantic mappings establish the correspondence between data elements in the sources and the concepts in the ontology. These components are expressed in the object-oriented deductive language F-Logic [15], which can be translated into Horn logic expressions that we use internally, or Web Ontology Language (OWL) and RuleML intended for the Semantic Web.

The core component in the mediation service module is the COIN mediator implemented in abductive constraint logic programming [13], where constraints are concurrently solved using Constraint Handling Rules (CHR) [7]. The mediator takes a user query and produces a set of mediated queries (MQs) that resolve semantic differences. This is accomplished by first 
translating the user query into a Datalog query and using the encoded knowledge to derive the MQs that incorporate necessary conversions from source contexts to receiver context. The query processor optimizes the MQs using a simple cost model and the information on source capabilities, obtains the data, performs the conversions, and returns the final datasets to the user.

Within the COIN approach, the users are not burdened with the diverse and changing semantics in data sources, all of which are recorded in the knowledge representation component and are automatically taken into account by the mediator. Adding or removing a data source is accomplished by adding and removing declarations, which does not require any changes to the mediator or query processor - they will use the new knowledge to produce the new correct conversion programs, as needed.

\subsection{Information Integration using COIN}

To apply COIN to the intelligence information integration scenario, none of the agencies need to change their current systems; they only need to record their context definitions by using the terms in a shared ontology. An excerpt of the ontology is shown in Figure 2.

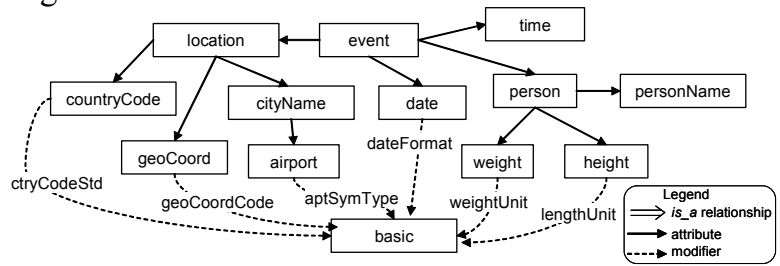

Figure 2. Excerpt of ontology

In the ontology, concepts, i.e., types, are in rectangular boxes. There is a special type called basic, which has no modifier and serves as the parent of all the other types. We do not show the is_a relationship between the type basic and the rest of the types to avoid cluttering the graph. The shared ontology is completely different from a data standard in that it only contains basic concepts and their relationships, which are much easier to agree on than the representations of the types that are usually specified in a data standard. For example, the ontology only states that a person has weight, keeping silent about in what unit the weight should be. With this ontology, each data source and receiver can define their local ontologies by specifying modifier values to obtain desired specializations to the common types, e.g., specializing "weight" to "weight in lbs." These specifications are called context definitions.
Table 3. Example contexts

\begin{tabular}{|l|l|l|l|l|}
\hline Modifier & USA context & UK context & NATO context & Analyst context \\
\hline dateFormat & $\mathrm{mm} / \mathrm{dd} / \mathrm{yyyy}$ & $\mathrm{dd} / \mathrm{mm} / \mathrm{yyyy}$ & dd.mm.yyyy & dd-mm-yyyy \\
\hline ctryCodeStd & FIPS & $\begin{array}{l}\text { ISO3166 } \\
\text { 2-alpha }\end{array}$ & $\begin{array}{l}\text { ISO3166 } \\
\text { 3-digit }\end{array}$ & $\begin{array}{l}\text { ISO3166 } \\
\text { 3-alpha }\end{array}$ \\
\hline aptSymType & IATA & ICAO & ICAO & IATA \\
\hline geoCoordCode & $\begin{array}{l}\text { MGRS- } \\
\text { WGS84 }\end{array}$ & BNG-OGB7 & $\begin{array}{l}\text { Geodetic- } \\
\text { WGS84 }\end{array}$ & UTM-WGS84 \\
\hline lengthUnit & inches & feet & $\mathrm{cm}$ & $\mathrm{m}$ \\
\hline weightUnit & pounds & stones & Kg & $\mathrm{kg}$ \\
\hline
\end{tabular}

Table 3 shows four example contexts that will be used later for a demonstration.

Both the ontology and the context definitions are declaratively defined and can be manipulated using graphic tools. Using the internal representation, the following F-Logic formula states that in context $c_{-} U S A$ the weight unit is $l b$ :

$\forall X$ : weight $\exists Y$ : basic $\vdash$

$$
X\left[\text { weightUnit }\left(c_{-} U S A\right) \rightarrow Y\right] \wedge Y\left[\text { value }\left(c_{-} U S A\right) \rightarrow ' l b^{\prime}\right] .
$$

The modifiers of a type are represented as methods of the type. The value method returns a value in the context specified by the parameter. This method is implemented by the mediator to compare the modifier values between the source context and the receiver context; if they are different, conversions are introduced to reconcile the differences.

Conversions are defined for each modifier between different modifier values; they are called component conversions. The mediator automatically composes a composite conversion using the component conversions defined for relevant modifiers to reconcile all semantic differences involved in a user query. In many practical cases, a component conversion can be parameterized to convert from any given context to any other given context for that modifier. For example, the following internal component conversion definition converts between any two contexts of weight units (a user-friendly interface can be used to define conversions):

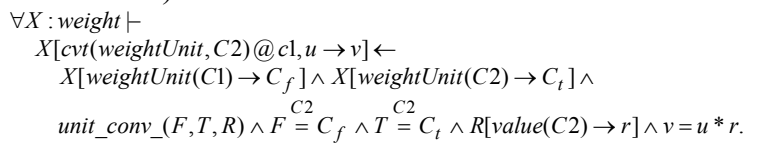

Once all contexts are defined and the component conversions for each modifier are specified, a receiver in any context can query any data source in other context as if they were in the same context. The mediator automatically recognizes context differences and dynamically composes a conversion using the component conversions.

We will demonstrate the key features of COIN using the intelligence information integration scenario. In Figure 3 we show the mediation of a query to two intelligence data sources, one in the USA context, the other in the UK context. In addition to converting data 
from different contexts into the desired context, the mediator also has explication tools such as reporting detected semantic differences and generating mediated queries as intensional answers to the original query. For example, when the receiver in the Analyst context issues the following query to combine data from two sources:

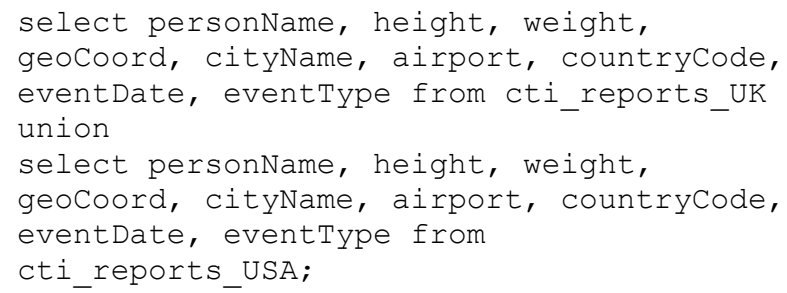

at the Conflict Detection stage, the mediator reports all detected semantic differences as shown in Table 4.

The first table in Table 4 displays the semantic difference between the UK context and the Analyst context, while the second table shows the differences between the USA context and the Analyst context. Comparing the detected differences here with those summarized in Table 3 indicates that all semantic differences are correctly identified. For example, weight is expressed in stones in the UK context while it is in $\mathrm{kg}$ in the Analyst context; because both the USA context and the Analyst context use the same airport code standard, the airport code difference shown in the first table does not appear in the second table. In fact, for the same query, if the desired context is USA context, the second table will be empty.

\section{Table 4. Semantic differences detected by mediator}

\begin{tabular}{|c|c|c|c|}
\hline SemanticType & Modifier & $\begin{array}{l}\text { Modifier value } \\
\text { in source contex }\end{array}$ & $\begin{array}{l}\text { Modifier value in } \\
t \text { target context }\end{array}$ \\
\hline eventDate & dateFmt & $\begin{array}{l}\text { c_UK : } \\
\text { European Style / }\end{array}$ & $\begin{array}{l}\text { c_Analyst : } \\
\text { American Style - }\end{array}$ \\
\hline countryCode & ctryCodeStd & $\begin{array}{l}\text { c_UK: } \\
\text { ISO3166A2 }\end{array}$ & $\begin{array}{l}\text { c_Analyst: } \\
\text { ISO3166A3 }\end{array}$ \\
\hline airportCode & aptSymType & c_UK : ICAO & c_Analyst : IATA \\
\hline geoCoord & geoCoordCode & $\begin{array}{l}\text { c_UK : BNG- } \\
\text { OGB7 }\end{array}$ & $\begin{array}{l}\text { c_Analyst : UTM } \\
\text { WGS84 }\end{array}$ \\
\hline Weight & weightUnit & c_UK : stone & c_Analyst : kg \\
\hline Height & lengthUnit & c UK : $\mathrm{ft}$ & c Analyst : $\mathrm{m}$ \\
\hline
\end{tabular}

(a) Differences between UK and Analysts contexts

\begin{tabular}{|l|l|l|l|}
\hline SemanticType & Modifier & $\begin{array}{l}\text { Modifier value in } \\
\text { source context }\end{array}$ & $\begin{array}{l}\text { Modifier value in } \\
\text { target context }\end{array}$ \\
\hline eventDate & dateFmt & $\begin{array}{l}\text { c_USA : } \\
\text { American Style / }\end{array}$ & $\begin{array}{l}\text { c_Analyst : } \\
\text { American Style - }\end{array}$ \\
\hline countryCode & ctryCodeStd & c_USA : FIPS & $\begin{array}{l}\text { c_Analyst : } \\
\text { ISO3166A3 }\end{array}$ \\
\hline geoCoord & geoCoordCode & $\begin{array}{l}\text { c_USA : MGRS- } \\
\text { WGGS84 }\end{array}$ & $\begin{array}{l}\text { c_Analyst : UTM- } \\
\text { WGS84 }\end{array}$ \\
\hline weight & weightUnit & c_USA : lb & c_Analyst : kg \\
\hline height & lengthUnit & c_USA : in & c_Analyst : m \\
\hline
\end{tabular}

(b) Differences between USA and Analyst contexts
The mediated query in the internal Datalog syntax is shown in Figure 3. All semantic differences shown in Table 4 are reconciled by the conversions automatically composed by the mediator. For example, the unit of measure difference for weight between UK context and the Analyst context is reconciled by using the unit_conv conversion function, which returns a conversion ratio (V15 indicated by a rectangle). The weight value in UK is V14 (indicated by an oval), which is multiplied by the conversion ratio to obtain V24 (in double-lined rectangle), which is $\mathrm{kg}$ as desired by the Analyst. Other semantic differences are reconciled similarly.

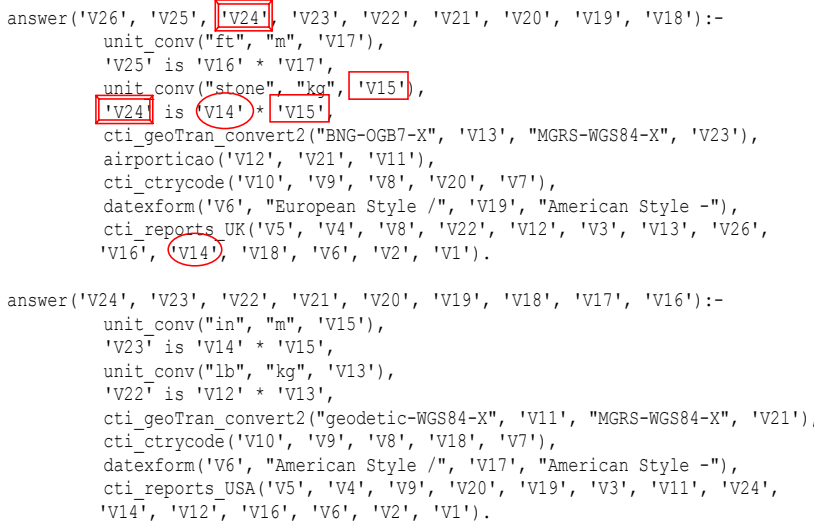

Figure 3. Mediated query

When the same query is issued by a receiver in other contexts, the appropriate mediated query will be generated accordingly. For example, Figure 4 shows the mediated query when the desired context is USA. Note that first sub-query now consists of necessary conversions between the UK context and USA context, e.g., weight conversion converts from stone to $\mathrm{lb}$. The second sub-query does not include any conversion at all, because the source is already in the receiver context.

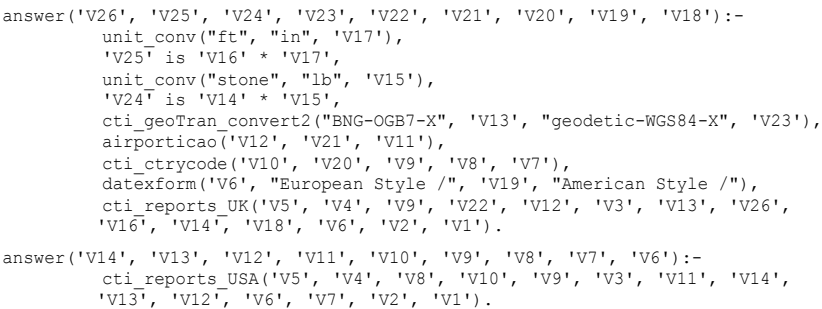

Figure 4. Mediated query when receiver is in USA context

We have shown with this example how the COIN approach overcomes the shortcomings of traditional approaches. That is, with COIN, the sources are not required to make any change or commit to any standard; they only need to record data semantics declaratively. Only a small number of component conversions need to be defined declaratively, which are used by the mediator to compose necessary 
conversions automatically. Changes in the sources can be accommodated by updating context definitions, no hand-written code need to be maintained. These features will be discussed further in the next section.

\section{Framework for Analyzing Adaptability, Extensibility, and Scalability}

The framework presented here is an extension to the preliminary work reported in [22]. It is motivated by [18] and based on an observation of necessary tasks involved to enable semantic interoperation. We categorize the tasks into three main categories:

- Knowledge acquisition: to acquire knowledge about all systems engaging in information exchange.

- Implementation: to encode the acquired knowledge, and implement necessary conversions as a set of instructions on how to reconcile semantic differences between systems.

- Execution: to fulfill a given information exchange task by determining and executing all necessary conversions to obtain data instances.

This task breakdown allows us to separate two aspects that need to be considered when evaluating semantic integration approaches. One concerns human efforts involved, the other concerns the performance of the software algorithm. Intuitively, the two aspects distinguish between "how hard humans have to work" and "how hard computers have to work" to achieve semantic interoperability. Tradeoffs can be made between "human efforts" and "computer efforts". For example, the global standard approach requires all systems implement the standard, in which case all systems are semantically interoperable by design. With this approach, most of the work is upfront human effort on developing and implementing the standard.

A set of criteria can be developed to evaluate different approaches for each task. By far the second task, i.e., implementation, is the most labor-intensive and requires significant amount of human efforts, so our framework will focus on assessing human efforts involved in carrying out the second task. The evaluation criteria consider three properties:

- Adaptability is the capability of accommodating changes, such as semantic changes within a data source with minimal effort.

- Extensibility is the capability of adding (or removing) data sources with minimal effort.

- Scalability refers to the capability of achieving and maintaining semantic interoperability with the amount of effort not growing dramatically with the number of participating sources and receivers.

Although a direct measurement of human efforts involved can be obtained through experiments, it will be costly to set up such experiments appropriately to reliably test different integration solutions. We take an alternative analytical approach that indirectly measures human efforts using the number of conversion programs to be manually developed, and maintained over time, as a proxy:

- Adaptability: number of conversions to be updated when data semantics changes.

- Extensibility: number of conversions to be added (or removed) when a source is added (or removed).

- Scalability: number of conversions needed for semantic interoperability among all sources and receivers.

The global standard approach eliminates the need for writing conversions, thus we will provide general discussions about its properties instead of using the proxy measurements. In the preceding discussions we used the term conversion quite loosely. To be more precise, we distinguish four types of conversion:

- a component conversion is defined for a modifier between two modifier values in the COIN approach; it reconciles one aspect of semantic differences of a single data type (e.g., a conversion that only reconciles differences in unit of weight);

- a compound conversion reconciles all aspects of semantic differences of a single data type (e.g., suppose that aside from weightUnit modifier, type weight also has a modifier to represent different scale factors in different contexts, a compound conversion reconciles differences in all aspects of weight, i.e., weight unit as well as scale factor);

- a composite conversion combines multiple component conversions to reconcile the semantic differences of all data types involved in a specific user query, which may access multiple data sources (e.g., a conversion that reconciles differences in weight, height, and geo-coordinate, supposing these data types are requested in a user query); and

- a comprehensive conversion reconciles the semantic differences of all data types in two sources or between a source and an interchange standard (e.g., a conversion that reconciles the differences in all data types of two systems, which could have dozens or even hundreds of data types).

Conceptually, a conversion of a latter type consists of multiple conversions of preceding types in the order shown above. Thus, when a conversion is implemented by writing code manually, on average it takes more human efforts for a latter type than for a previous type. In the next subsections, we illustrate the application of this evaluation framework by analyzing the integration approaches introduced in sections 3 and 4 . 


\subsection{Adaptability and Extensibility Analysis}

Since both adaptability and extensibility are concerned with changes, we use the term flexibility to collectively refer to the two properties in the ensuring discussion.

The Brute-force (BF) data conversion approach has the least flexibility. With $\mathrm{N}$ sources, a change in any source would affect $2(\mathrm{~N}-1)$ conversion programs, i.e., $\mathrm{N}-1$ conversion programs converting from the changing source to the other sources and vice versa. Adding or removing a source has similar effects.

This problem is somewhat reduced with the Interchange Standardization (IS) approach. But it still requires re-programming to handle changes, which can be tedious and error-prone. Furthermore, when the interchange standard is changed, all the $\mathrm{N}$ sources need to be updated to accommodate the change. All hardwiring approaches require the reconciliation of all semantic differences to be pre-determined and implemented in conversion programs. As a result, they lack flexibility.

The Global Data Standardization (GS) approach also lacks flexibility because any change requires agreement by all sources, which is difficult and extremely time consuming. Because it requires all systems to implement the changes, it sometimes causes disruption in operations.

In contrast, the ontology and context based COIN approach overcomes this problem. COIN has several distinctive features:

- It only requires that the individual contexts and individual conversions between a modifier's values (e.g., how to convert between weight units) be described declaratively. Thus it is flexible to accommodate changes because updating the declarations is much simpler than rewriting conversion programs (e.g., it is merely necessary to indicate that a source now reports in kilograms instead of stones).

- The customized conversion between any pair of sources (as many conversion programs as are needed) is composed automatically by the mediator using conversions of the relevant modifiers.

- COIN is able to generate all the conversions in BF, but without the burden of someone having to manually create and keep up-to-date all the pair-wise conversion programs.

- The COIN approach also avoids the multiple or unnecessary conversions that arise from the IS approach since the conversion programs that it generates only includes the minimally required conversions, including no conversions for certain (or all) modifiers, if that is appropriate.
As we will see from the next subsection, the COIN approach significantly reduces the number of predefined component conversions so that it can scale well when a large number of sources need to exchange information.

\subsection{Scalability Analysis}

As discussed earlier, our scalability analysis will focus on the number of conversions needed in each approach. The GS approach is scalable because it does not need any conversion at all. But it is often impossible to establish a global standard in large scale integration effort. We have informally discussed the scalability of the two other traditional approaches. We will summarize them followed by a detailed analysis on the scalability of the COIN approach.

Scalability of BF. With $\mathrm{N}$ data sources, the number of conversions for BF is $N(N-1)$, which is $O\left(N^{2}\right)$.

Explanation: Each source needs to perform translations with the other $N-1$ sources; there are $N$ sources, thus a total of $N(N-1)$ translations need to be in place to ensure pair-wise information exchange, which is $O\left(N^{2}\right)$.

Scalability of IS. With $\mathrm{N}$ data sources, the number of conversions for IS is $2 N$, which is $O(N)$.

Explanation: For each source there is a conversion to the standard and another conversion from the standard to the source. There are $\mathrm{N}$ sources, so the total number of conversions is $2 N=O(N)$.

Scalability of COIN. With $N$ data sources and an ontology that has $m$ modifiers with each having $n_{i}$ unique values, $i \in[1, m]$, the number of conversions for COIN is $O\left(m n_{k}^{2}\right)$, where $n_{k}=\max \left\{n_{i} \mid i \in[1, m]\right\}$; when $m$ is fixed, the number of conversions defined in COIN is $O\left(n_{k}^{2}\right)$.

Explanation: As seen earlier, conversions in COIN are defined for each modifier, not between pair-wise sources. Thus the number of conversions depends only on the variety of contexts, i.e., number of modifiers in the ontology and the number of distinct values of each modifier. In worst case, the number of conversions to be defined is $\sum_{i=1}^{m} n_{i}\left(n_{i}-1\right)$, where $n_{i}$ is the number of unique values of the $i^{\text {th }}$ modifier in the ontology, which is not to be confused with the number of sources; $m$ is the number of modifiers. This is because in worst case for each modifier, we need to write a conversion from a value to all the other values and vice versa, so the total number of conversions for the $i^{\text {th }}$ modifier is $n_{i}\left(n_{i^{-}}\right.$ 1). Let $n_{k}=\max \left(n_{l}, \ldots, n_{m}\right)$. When both $m$ and $n_{k}$ approach infinity, $\quad \sum_{i=1}^{m} n_{i}\left(n_{i}-1\right)=O\left(m n_{k}^{2}\right) ; \quad$ for $\forall m, \sum_{i=1}^{m} n_{i}\left(n_{i}-1\right)=O\left(n_{k}^{2}\right)$, as $n_{k} \rightarrow \infty$. 
However, in the intelligence information example, and in many practical cases, the conversion functions can be parameterized to convert between all values of a context modifier. For instance, the weight unit conversion given in Section 4 can convert between any two units of measure using the external relation unit_conv. The conversion functions for many other modifiers are also of this nature. Thus, only 6 of these parameterized conversion functions are necessary for converting between contexts that differ in weight, height, airport code, country code, geo-coordinate, and/or date format. The COIN approach can take advantage of these general functions because the overall conversion program between any two contexts is automatically generated.

When parameterization is impossible, we can still exploit certain relationships among component conversion functions. In cases where the set of component conversions are essentially a set of interrelated equations, COIN can generate undefined conversions using its symbolic equations solver $[5,6]$ to reduce the number of conversion component declarations needed. Thus the number of conversion definitions for a modifier can be reduced from $n(n-1)$ to $\mathrm{n}-1$, where $\mathrm{n}$ is the number of unique values of the modifier, leading to:

Scalability of COIN (parameterization and invertible conversion). When conversions can be parameterized, COIN requires $\mathrm{m}$ conversions. Otherwise, if the conversions are invertible functions,

COIN needs $\sum_{i=1}^{m}\left(n_{i}-1\right)$ conversions.

Furthermore, declaring the contexts can be simplified since contexts can be inherited with optional overriding in COIN. This significantly reduces the number of necessary declarations. For example, we can define a context $\mathrm{k}$ for a country because most agencies in the same country share the same context. If an agency in the country differs from the other agencies only with regard to say, weight unit, we can define its context as k' and specify only the particular weight unit in k'; by declaring k' as a sub-context of k, k' inherits all the other context definitions for context $k$. This keeps the size of the knowledge base compact when the number of sources grows. In addition, subtypes in the ontology inherit the modifiers and the conversion definitions of their parent types, which also helps keep the number of conversion component definitions small.

Table 5 summarizes the scalability of different approaches in terms of the number of conversions that need to be specified. Even in the worst case, the COIN approach requires significantly less conversions than the BF or IS approaches.
Table 5. Number of conversions to achieve semantic interoperability among 150 sources

\begin{tabular}{|l|l|l|}
\hline Approach & General case & The scenario \\
\hline $\begin{array}{l}\text { Brute Force } \\
\text { (BF) }\end{array}$ & $\begin{array}{l}N(N-1), N:=\text { number of sources } \\
\text { and receivers }\end{array}$ & 22,350 \\
\hline $\begin{array}{l}\text { Interchange } \\
\text { Standard (IS) }\end{array}$ & $\begin{array}{l}2 N, N:=\text { number of sources } \\
\text { and receivers }\end{array}$ & 300 \\
\hline $\begin{array}{l}\text { Context } \\
\text { Interchange } \\
\text { (COIN) }\end{array}$ & $\begin{array}{l}\text { 1) Worst case:, } \sum_{i=1}^{m} n_{i}\left(n_{i}-1\right) \\
n_{i}:=\text { number of unique values } \\
\text { of } t^{t h} \text { modifier, } m:=\text { number of } \\
\text { modifiers in ontology }\end{array}$ & 1) worst: 56 \\
2) $\sum_{i=1}^{m}\left(n_{i}-1\right)$ when equational & $\begin{array}{l}\text { 2) actual } \\
\text { relationships exist } \\
\text { number: } 6 \\
\text { parameterized if all conversions can be }\end{array}$ & \\
\hline
\end{tabular}

Recent research [21,23] extended COIN to represent and reason about semantics that change over time. For example, when comparing historic stock prices in different exchanges, some of them changed the currency assumed in the reporting (e.g., changed from reporting in French Francs to Euros). These temporal changes can be captured and the semantic differences at different times (in addition to between different sources) can be automatically recognized and reconciled at run time. With these advanced features and its flexibility and scalability, COIN is ideal for large scale information integration.

\section{Conclusion}

Integrating information from diverse heterogeneous systems is one of the key challenges today. Technological advances, such as web services and XML are reducing the cost of connecting software components both within the enterprise and across enterprise boundaries. At the same time the relative importance of achieving semantic interoperability has risen and alternative approaches to this problem may have vastly different long term costs and effectiveness. For example, it is not clear that the Army's success in limited scope integration of a dozen systems will scale to the demands of current efforts [1] to apply similar techniques to integration across the whole enterprise. Any viable solution must be flexible and scalable in reconciling semantic differences among information sources and must be able to support adaptation as requirements change.

As new solutions continue to emerge, it is important that practitioners and researchers understand the characteristics of different solution approaches. In this paper, we presented an evaluation framework and applied it to analyze several integration approaches. Our analysis shows that the COIN approach can efficiently handle a large number of semantic conflicts, 
and it is flexible and scalable to meet the evolving requirements.

We believe a systematic evaluation of integration solutions is an important area for future research. The current framework uses the measurement of human efforts involved in the implementation phase of information integration to evaluate the characteristics of an integration approach. Future research will develop mechanisms for assessing the amount of human efforts involved in knowledge acquisition phase.

Acknowledgements: This work has been supported, in part, by MITRE Corp., the MIT-MUST project, the Singapore-MIT Alliance, and Suruga Bank.

\section{References}

[1] Army Net-Centric Data Strategy (ANCDS) (2008). http://data.army.mil

[2] S. Bressan, C. Goh, N. Levina, S. Madnick, A. Shah, M. Siegel (2000) "Context Knowledge Representation and Reasoning in the Context Interchange System", Applied Intelligence: The International Journal of Artificial Intelligence, Neutral Networks, and Complex ProblemSolving Technologies, 12(2), pp. 165-179.

[3] D. Doughty (2004) "The Achilles' Heal of ServiceOriented Architectures", Business Integration Journal, September, 44-47

[4] H.T. El-Khatib, M.H. Williams, L.M. MacKinnon, D.H. Marwick (2000) "A Framework and Test-suite for Assessing Approaches to Resolving Heterogeneity in Distributed Databases", Information \& Software Technology, 42(7), 505-515.

[5] A. Firat, S.E. Madnick, B. Grosof (2002) "Financial Information Integration In the Presence of Equational Ontological Conflicts", Proceedings of the Workshop on Information Technology and Systems (WITS), Barcelona, Spain, December 14-15, pp. 211-216.

[6] A. Firat (2003) "Information Integration using Contextual Knowledge and Ontology Merging," $\mathrm{PhD}$ Thesis, MIT.

[7] T. Frühwirth (1998) "Theory and Practice of Constraint Handling Rules," J. of Logic Programming, 37, 95-138.

[8] M.P. Gallaher, A.C. O'Connor, J.L. Dettbarn, and L.T. Gilday (2004) "Cost Analysis of Inadequate Interoperability in the U.S. Capital Facilities Industry", GCR 04-867, NIST

[9] H. Greene \& R. Mendoza (2005) "Lessons Learned From Developing the ABCS 6.4 Solution.” Defense AR Journal (April-July 2005): 177-189.

[10] C.H. Goh (1997) "Representing and Reasoning about Semantic Conflicts in Heterogeneous Information Systems", PhD Thesis, MIT.
[11] C.H. Goh, S. Bressan, S. Madnick, M. Siegel (1999) "Context Interchange: New Features and Formalisms for the Intelligent Integration of Information", ACM Trans. on Information Systems (TOIS), 13(3), 270-293.

[12] M. Hammer, M. Stonebraker, O. Topsakal (2005) "THALIA: Test Harness for the Assessment of Legacy Information Integration Approaches", ICDE'05, 485486.

[13] A.C. Kakas, A. Michael, and C. Mourlas (2000) "ACLP: Integrating Abduction and Constraint Solving," Journal of Logic Programming, 44, pp. 129-177.

[14] V. Kashyap, A.P. Sheth (2000) Information Brokering Across Heterogeneous Digital Data: A Metadata-based Approach, Springer.

[15] M. Kiffer, G. Laussen, J. Wu (1995) "Logic Foundations of Object-Oriented and Frame-based Languages", J. ACM, 42(4), pp. 741-843.

[16] R. Miller, M.A. Malloy, E. Masek (2003) "Transforming Tactical Messaging: Exploiting Web Information Standards for Interoperability", Intercom, 44(1), 50-51.

[17] National Research Council (2002) "Making the Nation Safer: The Role of Science and Technology in Countering Terrorism", The National Academies Press.

[18] A. Rosenthal, L. Seligman, S. Renner, F. Manola (2001) "Data Integration Needs an Industrial Revolution", Int'l Workshop on Foundations of Models for Info. Integration (FMII'01), Viterbo, Intaly.

[19] A. Rosenthal, L. Seligman, S. Renner (2004) "From Semantic Integration to Semantics Management: Case Studies and a Way Forward", ACM SIGMOD Record, 33(4), 44-50.

[20] H. Wache, T. Vogele, U. Visser, H. Stuckenschmidt, G. Schuster, H. Neumann, S. Hubner (2001) "OntologyBased Integration of Information - A Survey of Existing Approaches", Proceedings of the IJCAI-01 Workshop on Ontologies and Information Sharing, Seattle, USA, August 4-5.

[21] H. Zhu, S. Madnick, M. Siegel (2004) "Reasoning about Temporal Context using Ontology and Abductive Constraint Logic Programming", Workshop on Principles and Practices of Semantic Web Reasoning (PPSWR04), Saint Malo, France, Sep 8-9, in LNCS 3208, 90-101.

[22] H. Zhu, S.E. Madnick (2004) "Context Interchange as a Scalable Solution to Interoperating Amongst Heterogeneous Dynamic Service", 3rd Workshop on EBusiness, December 11, 2004, Washington, D.C., 150161.

[23] H. Zhu (2005) "Effective Information Integration and Reutilization: Solutions to Technological Deficiency and Legal Uncertainty", PhD Thesis, MIT. 